\title{
Relações celulares na abordagem e prognóstico do câncer de mama: revisão integrativa da literatura
}

\author{
Cellular Ratios in the Approach and Prognosis of Breast Cancer: an integrated literature review \\ Relaciones celulares en el abordaje y pronóstico del cáncer de mama: revisión bibliográfica
}

integradora

Recebido: 15/10/2021 | Revisado: 23/10/2021 | Aceito: 25/10/2021 | Publicado: 29/10/2021

\author{
Caio André de Oliveira Alcântara \\ ORCID: https://orcid.org/0000-0002-7526-5586 \\ Centro Universitário São Lucas, Brasil \\ E-mail:caioandre10@hotmail.com \\ Camilla Vitória Querino do Carmo \\ ORCID: https://orcid.org/0000-0001-8512-2776 \\ Centro Universitário São Lucas, Brasil \\ E-mail: camillavqc@gmail.com \\ Gabriel Guimarães Cunha Silva \\ ORCID: https://orcid.org/0000-0003-0535-2577 \\ Centro Universitário São Lucas, Brasil \\ gabrielguimaraesgcs@gmail.com \\ Karol Stheffani Mollinedo Pereira \\ ORCID: https://orcid.org/0000-0002-5562-510X \\ Centro Universitário São Lucas, Brasil \\ E-mail: karolstheffani@icloud.com \\ Yasmin Nogueira de Sousa \\ ORCID: https://orcid.org/0000-0002-6478-1462 \\ Centro Universitário São Lucas, Brasil \\ E-mail: nogueirayasmin21@gmail.com \\ Douglas Reis Abdalla \\ ORCID: https://orcid.org/0000-0002-6971-1201 \\ Universidade de Uberaba, Brasil \\ Faculdade de Talentos Humanos, Brasi \\ E-mail: drabdalla@facthus.edu.br
}

\begin{abstract}
Resumo
O câncer de mama, é atualmente a neoplasia que mais acomete mulheres em todo o mundo, e tendo em vista que o surgimento dessa patologia se dá pela soma de diversos fatores tais como a predisposição genética, torna-se complexo a elaboração de prognósticos da doença. Dessa forma, é necessário buscar meios embasados em pesquisas científicas que auxiliem nesse contexto. Esse estudo, teve como objetivo discutir a conformidade existente entre as relações celulares e seus significados no câncer de mama. Para tanto, foi realizada revisão Integrativa da literatura na base de dados PubMed das produções dos últimos 10 anos. Os descritores utilizados foram: "relação neutrófilo linfócito", "relação monócito linfócito", "câncer de mama" e "prognóstico", os quais foram conjugados para delimitação da busca. Foram selecionados 6 artigos ao final das etapas de busca. Sendo os anos de 2018 e 2020 os mais prevalentes com 33,3\% das publicações cada, seguido pelo ano de 2019 e 2021 com 16,6\% das publicações cada. Em relação ao país de origem dos estudos, 2 artigos foram realizados na Polônia (33,3\%), e em cada um desses países, sendo eles China, Espanha, Bélgica e República da Coréia foram publicados um artigo respectivamente (16,6\%). Evidências mostraram que pacientes com NLR mais elevados obtiveram tempo de sobrevida menor, por outro lado, pacientes com valores mais altos de LMR possuíram melhor prognóstico que as demais relações. Portanto, vale ressaltar a importância das relações celulares a serem investigadas em pacientes com câncer de mama com o intuito de otimizar o prognóstico e, consequentemente, buscar os melhores métodos terapêuticos para elevar o tempo de sobrevida dos neoplásicos.
\end{abstract}

Palavras-chave: Câncer de mama; Relações celulares; Prognóstico; Biomarcadores inflamatórios; Sobrevida.

\begin{abstract}
Breast cancer is currently the cancer that most affects women worldwide, and taking into account that the appearance of this pathology is the sum of several factors such as genetic predisposition, it becomes complex the elaboration of disease prognoses. Thus, it is necessary to seek means based on scientific research that can help in this context. This study aimed to discuss the existing conformity between cellular relations and their meanings in breast cancer. For this,
\end{abstract}


an integrative literature review was carried out in the PubMed database of the productions from the last 10 years. The descriptors used were: "neutrophil lymphocyte ratio", "monocyte lymphocyte ratio", "breast cancer" and "prognosis", which were combined to delimit the search. Six articles were selected at the end of the search steps. Being the years 2018 and 2020 the most prevalent with $33.3 \%$ of publications each, followed by the year 2019 and 2021 with $16.6 \%$ of publications each. Regarding the country of origin of the studies, 2 articles were conducted in Poland (33.3\%), and in each of these countries, being China, Spain, Belgium, and the Republic of Korea, one article was published respectively (16.6\%). Evidence showed that patients with higher NLR obtained shorter survival time, on the other hand, patients with higher values of LMR had better prognosis than the other relations. Therefore, it is worth emphasizing the importance of cellular ratios to be investigated in patients with breast cancer in order to optimize the prognosis and, consequently, to seek the best therapeutic methods to raise the survival time of neoplastics.

Keywords: Breast cancer; Cellular ratios; Prognosis; Inflammatory biomarkers; Survival.

\section{Resumen}

El cáncer de mama es en la actualidad la neoplasia que más afecta a las mujeres en todo el mundo, y teniendo en cuenta que la aparición de esta patología se debe a la suma de diversos factores como la predisposición genética, la elaboración de los pronósticos de la enfermedad se hace compleja. Por ello, es necesario buscar medios basados en la investigación científica para ayudar en este contexto. Este estudio tenía como objetivo discutir la conformidad entre las relaciones celulares existentes y sus significados en el cáncer de mama. Para ello, se realizó una revisión bibliográfica integradora en la base de datos PubMed de las producciones de los últimos 10 años. Los descriptores utilizados fueron: "relación neutrófilo-linfocito", "relación monocito-linfocito", "cáncer de mama" y "pronóstico", que se conjugaron para delimitar la búsqueda. Al final de las etapas de búsqueda se seleccionaron seis artículos. Siendo los años 2018 y 2020 los más prevalentes con un 33,3\% de publicaciones cada uno, seguidos del año 2019 y 2021 con un $16,6 \%$ de publicaciones cada uno. En cuanto al país de origen de los estudios, 2 artículos se realizaron en Polonia $(33,3 \%)$, y en cada uno de estos países, siendo China, España, Bélgica y la República de Corea se publicó un artículo respectivamente $(16,6 \%)$. Las pruebas mostraron que los pacientes con un NLR más alto obtuvieron un tiempo de supervivencia más corto, por otro lado, los pacientes con valores más altos de LMR tuvieron un mejor pronóstico que las otras relaciones. Por lo tanto, cabe destacar la importancia de los ratios celulares a investigar en pacientes con cáncer de mama para optimizar el pronóstico y, en consecuencia, buscar los mejores métodos terapéuticos para aumentar el tiempo de supervivencia de las pacientes neoplásicas.

Palabras clave: Cáncer de mama; Relaciones celulares; Pronóstico; Biomarcadores inflamatorios; Supervivencia.

\section{Introdução}

A neoplasia é caracterizada por uma série de eventos em cadeia, que engloba alterações genéticas causadas por fatores ambientais, crescimento celular desordenado e erros nas reações imunológicas como a falha da morte celular programada, causada por moléculas antiapoptóticas que bloqueiam esse evento fisiológico, resultando em processos inflamatórios crônicos e promovendo o desenvolvimento e crescimento de tumores. Assim sendo, um dos principais tipos de neoplasia no Brasil e no mundo é o câncer de mama, que em $90 \%$ dos casos tem como precursor dos eventos já citados os hábitos de vida como: o sedentarismo, alimentação desequilibrada, alterações hormonais que podem ocorrer por gestações e uso de pílulas anticoncepcionais; e nos $10 \%$ restante têm-se a predisposição genética como vilã (Luchs \& Pantaleão, 2010; Inumaru et al., 2011; Faria et al., 2021).

Para corroborar a incidência dessa patologia no Brasil, o Instituto Nacional de câncer (INCA), divulgou dados do levantamento de 2020, no qual o câncer de mama aparece como o mais prevalente nas mulheres, com exceção da neoplasia de pele não melanoma, totalizando 66.280 novos casos, um total de 29,2\% das diferentes neoplasias diagnosticadas no gênero feminino no ano em questão (INCA, 2020).

Outrossim, o Câncer de Mama é uma doença que em sua fase inicial é geralmente assintomática, o que dificulta um possível diagnóstico precoce (Salimena et al, 2012). No entanto, há relatos que em fases iniciais esse câncer pode apresentar normalmente alguns sinais e sintomas, como: nódulo, fixo e geralmente indolor; pele da mama com aspecto de "casca de laranja"; alterações no mamilo; pequenos nódulos nas axilas ou pescoço; saída espontânea de líquido hemorrágico pelos mamilos (INCA, 2020).

Diante disso, o principal método de diagnóstico do câncer de mama é a mamografia, um dos métodos mais eficazes e mais creditáveis para detecção de lesões subclínicas na fase inicial da doença, aumentando as possibilidades de um melhor 
prognóstico. (Godinho \& Koch, 2004). Além desse, tem-se a ultrassonografia que é indicada quando há alterações na mamografia, facilitando a detecção de achados em mamas densas e auxiliando na coleta da biópsia (Nastri et al., 2011). Por fim, citado anteriormente temos a biópsia, importante método de diagnóstico precoce, que possui duas técnicas mais famosas, a biópsia por agulha grossa ou core biopsy, na qual possibilita o estudo histopatológico do tumor, e a punção biópsia aspirativa, que viabiliza o estudo citopatológico do material coletado (Ribeiro-Silva, 2012).

Pacientes possuem determinadas individualidades genéticas que influenciam os fatores de risco e/ou o prognóstico do câncer de mama, (onde ou na qual) a alta capacidade de sofrer metástase aliada a uma evolução imprevisível desse tipo de câncer resulta num prognóstico variável (Batschauer, 2009). Ademais, esse prognóstico é determinado por indicadores do curso clínico, os fatores prognósticos, que abordam o risco de recidiva ou morte, além de facilitarem uma análise específica do comportamento do tumor mediante sua evolução e, assim, contribuem para uma terapia adjuvante (Diana et al., 2004; Azambuja, 2007; Guerra, 2007; Batschauer, 2009).

Sendo assim, esses fatores prognósticos são determinados com base no tumor e no paciente. De acordo com o tumor têm-se, tipo histológico, grau de diferenciação, tamanho, presença de receptores hormonais e invasão linfonodal; já quanto o paciente tem-se, idade ao diagnóstico, histórico familiar, índice de massa corporal (IMC) e algumas características genéticas ainda pouco definidas (Azambuja, 2007; Batschauer, 2009).

Estudos têm demonstrado os impactos do processo inflamatório na tumorigênese do câncer (CA) de mama, desde o início, progressão, metástases e resistência ao tratamento (Abdalla et al., 2015; Bianco et al., 2017). Biomarcadores inflamatórios, como a razão linfocitária neutrofílica (NLR), a razão linfocitária plaquetária (PLR) e a razão linfocitária monocitária (LMR), estão sendo utilizados, rotineiramente, para avaliação do valor prognóstico em diferentes doenças (Araújo et al., 2021), mas também em estágios do câncer de mama. A NLR é definida pela contagem de neutrófilos dividida pela contagem de linfócitos. Altos valores de NLR estão associados a um pior prognóstico em vários tipos de tumores sólidos, enquanto baixos valores estão relacionados a um menor risco de reincidência e a uma maior taxa de sobrevivência geral (SO). A PLR é definida pela contagem de plaquetas dividida pela contagem de linfócitos. Altos valores de PLR estão associados a pior prognóstico e maior risco de metástases em pacientes com CA de mama. Por fim, a LMR é definida pela contagem de linfócitos dividida pela contagem de monócitos. Alguns estudos apontam que altos valores de LMR podem estar relacionados a um prognóstico positivo quanto à sobrevida livre de doença em pacientes com câncer metastático. (Huszno \& Koloska, 2019; Van Berckelaer, et al., 2020).

Ainda que pesquisas apontem uma forte correlação entre a inflamação sistêmica e o pior valor prognóstico no CA de mama, os mecanismos pelo qual isso ocorre ainda não foram bem elucidados. Esse presente estudo, tem como objetivo principal discutir a conformidade existente entre as relações NLR, LMR e PLR e seus significados no câncer de mama.

\section{Metodologia}

No presente estudo foi conduzida uma revisão integrativa, que consiste em uma pesquisa que permite a partir de evidências a avaliação, síntese e conhecimento acerca de um fenômeno, objetivando produzir uma visão geral de conceitos complexos, teorias ou problemas de saúde relevantes a partir de estudos pré-existentes, possibilitando a proposição de intervenção (Souza; Silva \& Carvalho, 2010).

Para a seleção dos artigos, foram conduzidas 6 etapas metodológicas, quais sejam: 1. elaboração da questão norteadora ou hipótese da pesquisa, ou seja, identificou-se o problema, apresentou-se o mecanismo de busca e os descritores ou palavras chave; 2. estabelecimento dos critérios de inclusão e exclusão dos artigos a serem selecionados para composição da amostra; 3. leitura exploratória dos títulos e resumos dos artigos para pré-seleção; 4. leitura analítica dos artigos a fim de compilar, analisar e categorizar as informações; 5. interpretação dos resultados. 6. síntese seguida da apresentação dos 
resultados identificados, que permeiam a questão norteadora (Botelho; Cunha \& Macedo, 2011).

Portanto, neste estudo optou-se por realizar busca sobre os conceitos: Monocyte to lymphocyte ratio, monocytelymphocyte ratio, neutrophil to lymphocyte ratio, neutrophil-lymphocyte ratio, breast câncer, breast tumor. A partir desses conceitos, definiu-se a questão norteadora: Qual a relação das células pró-inflamatórias com os linfócitos no prognóstico de pacientes com câncer de mama?

Após a formulação da questão a ser pesquisada, foi realizado um levantamento bibliográfico na plataforma PubMed. O levantamento do estudo ocorreu entre março e abril de 2021. E a seleção dos textos procedeu com as buscas na plataforma, utilizando os filtros nela disponível para textos publicados entre 2010 e 2021. Para seleção das publicações, foram adotados os seguintes critérios de inclusão: artigos científicos, publicados no idioma Inglês, entre os anos de 2010 a 2020, disponíveis online e gratuitamente na íntegra. Foram excluídos os artigos sem resumo na base de dados ou incompletos, editoriais, cartas ao editor, estudos reflexivos, revisões sistemáticas ou integrativas de literatura.

Após a definição da questão norteadora, localização e seleção dos artigos, foram identificadas 19 publicações potencialmente elegíveis para serem incluídas nessa revisão. Após a aplicação dos critérios de inclusão e exclusão a amostra foi composta por 19 publicações, foram analisados os resumos de 19 registros, para verificar se atenderiam os critérios de elegibilidade e se responderiam à pergunta que norteia esta revisão, assim excluiu-se 9 registros e somente 10 foram analisados na íntegra para confirmar a elegibilidade para a síntese quantitativa e análise dos dados conforme o fluxograma de seleção (Figura 1).

Figura 1: Fluxograma de Seleção das Publicações.

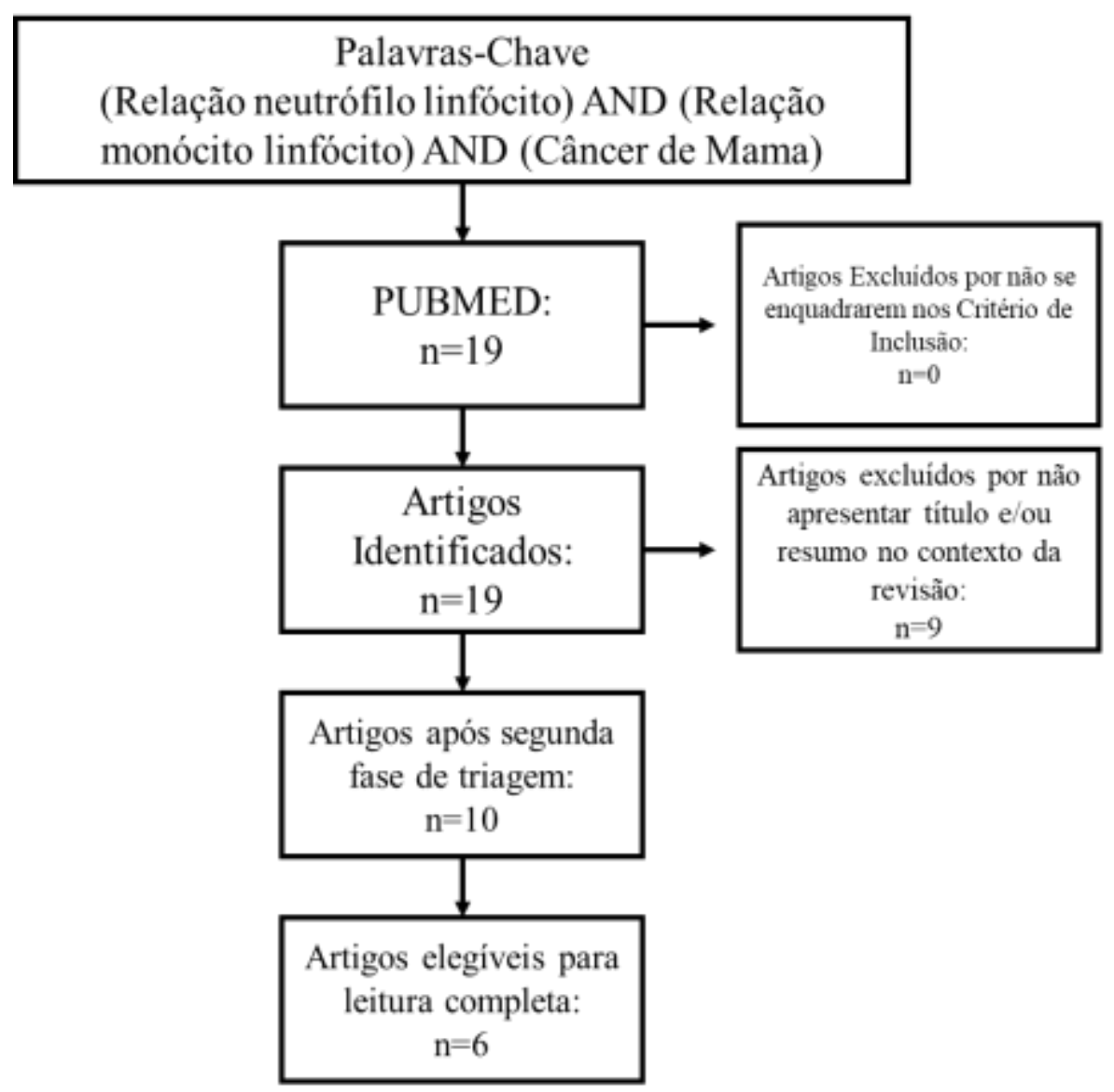

Fonte: Autores (2021). 


\section{Resultados e Discussão}

No espaço de tempo delimitado para a realização deste estudo (2011-2021) foram encontradas e analisadas 6 publicações, sumarizadas na Tabela 1. Em 2019 e 2021 foram publicados 1 artigos $(16,6 \%)$ em cada ano respectivamente. Nos anos de 2018 e 2020 constam 2 publicações em cada ano $(33,3 \%)$.

De acordo com a metodologia dos trabalhos selecionados os tipos de estudo eram pesquisas observacionais, longitudinais e coorte utilizando as relações celulares como base para análise de diversas variáveis.

As publicações resultaram de diferentes revistas sendo: Oncology Letters, Springer Nature, Dove Press Journal, The Breast, Plos One, Oncology. Analisando os locais de estudo 2 artigos foram realizados na Polônia (33,3\%), e em cada um desses países, sendo eles China, Espanha, Bélgica e República da Coréia foram publicados um artigo respectivamente (16,6\%).

Tabela 1: Artigos levantados na base de dados Pubmed acerca dos artigos relacionando câncer de mama e o significado das relações celulares.

\begin{tabular}{|c|c|c|c|}
\hline Referência & Ano de publicação & Tipo de Estudo & Objetivos do estudo \\
\hline Cho et al & 2018 & Observacional & $\begin{array}{l}\text { O objetivo foi verificar a relação entre os índices inflamatórios pré- } \\
\text { tratamento (ou seja, o NRL, PLR e LMR) e o prognósticos de } \\
\text { pacientes com câncer de mama e investigar qual marcador é mais útil } \\
\text { como um fator prognóstico. }\end{array}$ \\
\hline Losada et al & 2019 & $\begin{array}{l}\text { Observacional } \\
\text { longitudinal }\end{array}$ & $\begin{array}{c}\text { No presente estudo, examinamos essas células inflamatórias } \\
\text { periféricas em pacientes com câncer de mama maior ou igual a } 65 \\
\text { anos e correlacionamos nossas descobertas com sobrevida livre de } \\
\text { doença (SLD) e sobrevida geral (SG). }\end{array}$ \\
\hline $\begin{array}{l}\text { Huszno \& } \\
\text { Kolosza }\end{array}$ & 2019 & Observacional & $\begin{array}{l}\text { O objetivo do presente estudo foi avaliar o sangue, o NRL, PLR e } \\
\text { MLR como fatores prognósticos em pacientes com câncer de mama. }\end{array}$ \\
\hline Huszno et al & 2020 & Observacional & $\begin{array}{l}\text { O objetivo do presente estudo foi avaliar o NLR, PLR e MLR } \\
\text { sanguíneos como fatores prognósticos para pacientes com CM do sexo } \\
\text { masculino. }\end{array}$ \\
\hline $\begin{array}{l}\text { Van Berckelaer } \\
\text { et al }\end{array}$ & 2020 & $\begin{array}{l}\text { Observacional } \\
\text { Longitudinal }\end{array}$ & $\begin{array}{c}\text { Neste estudo, investigamos o papel prognóstico dos marcadores de } \\
\text { inflamação periférica em IBC, fizemos uma comparação com nIBC e } \\
\text { exploramos a relação entre esses marcadores sistêmicos e a resposta } \\
\text { imune local. }\end{array}$ \\
\hline Ma \& Chen & 2021 & $\begin{array}{l}\text { Observacional } \\
\text { Longitudinal }\end{array}$ & $\begin{array}{c}\text { Dados clínicos e de acompanhamento de } 203 \text { pacientes com câncer de } \\
\text { mama foram analisados para investigar o valor do NLR, PLR e LMR } \\
\text { do sangue periférico na previsão da recorrência pós-operatória de } \\
\text { câncer de mama e para fornecer referências apropriadas para } \\
\text { aplicabilidade clínica. }\end{array}$ \\
\hline
\end{tabular}

Fonte: Autores (2021).

No estudo de Cho e Cols (2018), buscaram demonstrar que alguns marcadores inflamatórios sistêmicos derivados de células do sangue periférico, como NLR, PLR e LMR podem ser utilizados como marcadores de prognóstico em vários tipos de malignidade, além de associá-los ao tempo de sobrevida em pacientes com Câncer de Mama. Para isso, avaliaram 661 pacientes submetidos a cirurgia para tratamento de CA invasivo entre 2003 e 2011, onde três deles eram do sexo masculino, com faixa etária no momento de diagnóstico de 52,7 $\pm 11,5$ anos. Os autores evidenciaram que 432 pacientes $(65,4 \%)$ tiveram um NLR alto, 82 pacientes (12,4\%) tiveram um PLR alto e 619 pacientes $(93,6 \%)$ tiveram um alto LMR. Desta forma, as relações celulares são marcadores prognósticos independentes, destaque para PLR previu com precisão a probabilidade de sobrevida individualizada no câncer de mama.

No estudo de Losada e Cols (2019), buscaram a importância das relações celulares NLR, LMR e PLR na sobrevida de pacientes com câncer de mama. Foram avaliadas 113 mulheres com 65 anos ou mais, as quais foram analisadas por um período médio de 48 meses. Entre as variáveis analisadas em relação a sobrevida, temos: idade, tipo de tratamento recebido pela paciente anteriormente, as relações celulares, tipo de câncer, envolvimento linfodonal. Como resultado, evidenciou-se que o 
subgrupo de 25 pacientes com o tipo de tumor Triplo Negativo (TNBC), no que tange a sobrevida livre de doença (SLD) $(\mathrm{p}<0,001)$ e sobrevida global $(\mathrm{SG})(\mathrm{p}=0,03)$ foram ambos maiores em pacientes com baixo NLR, e SLD $(\mathrm{p}=0,03)$ foi também maior em pacientes com baixa PLR. Em outros 69 pacientes que foram analisados por menos de 3 anos, SLD (p=0,05) foi maior em pacientes com PLR baixo (menor ou igual a 150) e pacientes vivos mas com doença recorrente ( $\mathrm{P}=0,05)$ possuíam PLR alto (> 150). PLR acima de 150 esteve relacionado com maiores tumores. Já os subtipos de tumores Luminal A, Luminal B e HER2 ( $\mathrm{p}=0,02$ ), evidenciaram NLR mais baixos (NLR menor ou igual a 3,33) que os pacientes com subtipo TNBC.

No estudo de Huszno e Kolosza (2019), buscaram avaliar o valor prognóstico da NLR, PLR e LMR em pacientes com câncer de mama. Para isso foi elaborado o delineamento em que durante 2005 e 2018, 436 pacientes com câncer de mama, na faixa etária de 25,2 a 78,3 anos, foram randomizados para exames de acompanhamento clínico a cada três meses nos primeiros dois anos, depois a cada seis meses até o quinto ano após o diagnóstico e, em seguida, todos os anos. As variáveis analisadas mais relevantes foram a idade, grau do tumor, estado de estrogênio, subtipo molecular, metástases de gânglios linfáticos e taxa de sobrevida. Pacientes com NLR>2,65 eram frequentemente mais jovens $(\mathrm{p}=0,021)$ e tinham uma taxa de sobrevida de 5 anos menor quando comparado com o subgrupo NLR $<2,65$, particularmente naquelas pacientes com câncer de mama tri-negativo $(\mathrm{p}=0,034)$, em pacientes com tumores com status $\mathrm{ER}(-)(\mathrm{p}=0,018)$ ou com um grau de tumor mais alto $(\mathrm{p}=0,020)$. Pacientes com um PLR>190,9 tinham pior taxa de sobrevida, especialmente nos subgrupos com TNBC ( $=0,032)$, status ER(-) $(\mathrm{p}=0,002)$ ou tumores com um grau histológico mais alto $(\mathrm{p}=0,002)$. Assim, pacientes que apresentam níveis altos de NLR, PLR e LMR possuem um pior prognóstico no câncer de mama.

No estudo de Huszno et al. (2020), buscaram avaliar as relações NLR, PLR e LMR como fatores prognósticos em pacientes do sexo masculino que apresentem Câncer de Mama. Para isso foi elaborado o delineamento, na qual realizou-se uma análise retrospectiva entre 2005 e 2018, com 38 homens portadores de câncer de mama, na faixa etária de 31 a 84 anos, sendo esses randomizados para exames de acompanhamento clínico a cada três meses nos primeiros dois anos, depois a cada seis meses até o quinto ano após o diagnóstico e a cada ano a partir de então. Vale ressaltar que, as variáveis analisadas que possuem destaque são médias de idade, histórico familiar de câncer e histórico de câncer de mama, idade $\leq 56$ anos, níveis de hemoglobina $\leq 15.9 \mathrm{~g} / \mathrm{dL}$, contagem de linfócitos inferiores $\leq 1.82 \times 103 / \mu \mathrm{L}$. Diante disso, pacientes com média de idade de 69 anos apresentam NLR > 2,74 ( $\mathrm{p}=0.005)$ e aqueles com média de 77 anos apresentam PLR $>169.1(\mathrm{p}=0.0003)$. Pacientes com uma PLR elevada (> 169,1) foram associados a uma pior taxa de sobrevida, assim como naquele grupo com LMRs (> 0,30). Assim, os presentes resultados revelam que um LMR elevado (>0,30), PLR (> 169,1) ou NLR $(>2,74)$ estão associados a uma baixa sobrevida geral de pacientes com câncer de mama do sexo masculino.

No estudo de Van Berckelaer et al, (2020), procurou-se avaliar o impacto que a resposta imune periférica e alguns biomarcadores sanguíneos, como a relação neutrófilos-linfócitos (NRL) tem sobre o valor prognóstico dos pacientes com Câncer de Mama. Para que isso fosse possível, realizaram o delineamento, no qual utilizaram prontuários de pacientes diagnosticados com câncer de mama inflamatório, de dois hospitais, sendo o primeiro com prontuários datados de 1997 a 2017, e o segundo de 2006 a 2017, totalizando 127 pacientes. O estudo permitiu relacionar as relações celulares com a sobrevida livre de recorrência da doença (SLRD), Sobrevida distante livre de metástases (SLM) e SG, bem como a prevalência de determinados tipos de câncer que utilizam as relações celulares como fator determinante para sua variabilidade. Como resultado, notaram que NLR foi maior em câncer inflamatório em relação ao câncer em estágio inicial (2,70 e 2,14 respectivamente), com valor de $\mathrm{P}=0,006$. Na população total de pacientes com IBC, pacientes com alto nível de NLR $(>4,0)$ tiveram um resultado pior em comparação com um NLR inferior. A sobrevida mediana para este marcador foi de 5,54 anos e no grupo com altos valores de NLR a sobrevida foi de 2,17 anos. O PLR foi maior em pacientes com doença metastática (PLR medio $=183)$ com $\mathrm{P}=0,01)$ mas não houve diferença entre $\mathrm{mIBC}$ e mBC para essa relação celular. Um PLR inferior (210) foi correlacionado com melhor SLD e SLM ( $\mathrm{p}=0,03$ e p=0,018 respectivamente). O LMR foi maior no nIBC estágio LABC (LMR 
médio: 4,56) em contraste com IBC (LMR médio: 3,29, p <0,001) . O número de monócitos em nIBC LABC também foi significativamente menor em comparação com $\operatorname{IBC}(p=0,001)$ e eBC $(p=0,002)$. Na população total de pacientes com IBC, pacientes com alto nível de NLR $(>4,0)$ tiveram um resultado pior em comparação com um NLR inferior. Assim, é possível concluir que tanto IBC quanto nIBC provocam a mesmo resposta imune periférica e a PLR e NLR devem ser vistas como marcadores de doença extensa. No entanto, os pacientes com um alto NLR $(>4,0)$ têm um resultado pior em IBC, e um alto PLR está associado com um RFS adverso independente do estágio da doença ou subtipo molecular.

No estudo de Ma e Chen (2021), avaliaram o efeito da quimioterapia neoadjuvantes e a sobrevida livre geral utilizando como preditores as relações celulares NLR, PLR e LMR. A pesquisa foi realizada por um periodo médio de 31 meses, com 203 mulheres entre 17 e 71 anos com câncer de mama. As quais foram divididas em dois grupos, o primeiro incluiu as pacientes que tiveram metastases de orgãos locais ou distantes após a operação, e o segundo incluiram se pacientes que não obtiveram recidivas. Entre as principais variáveis utilizadas temos: idade, ciclo menstrual, história familiar, estágio T, metástase de linfonodo, subtipos de câncer de mama, radioterapia, quimioterapia neoadjuvante, inflamação e os marcadores NLR, PLR e LMR. Os níveis de NLR e PLR no grupo não recorrente foram considerados significativamente mais baixos do que aqueles no grupo recorrente ( $\mathrm{p}<0,001)$. No estudo feito foi possível observar que em relação a sobrevida dos pacientes livre de câncer $(\mathrm{p}=0,001)$, a maioria dos pacientes com melhor prognóstico possuem um LMR >6.2, a NLR e PLR não mostraram correlação significativa com o prognóstico da doença. Uma elevada NLR e PLR indicaram um SLD pobre após ressecção cirúrgica. Uma baixa LMR indicou um SLD pobre. Os demais resultados analisados não foram relevantes, pois de acordo com os gráficos $\mathrm{P}>0,05$ foram deixando os dados inelegíveis para utilização na revisão bibliográfica. Os resultados revelaram que baixo LMR indica mau prognóstico. Embora NLR e PLR não sejam indicadores de prognóstico de SLD em análise multivariada, e os valores de NLR e PLR foram maiores na recorrência e grupos de metástases e relacionadas ao tamanho do tumor, sugerindo que NLR e PLR têm certo significado clínico. Dentro geral, LMR é um marcador de melhor prognóstico do que NLR e PLR na previsão do prognóstico do câncer de mama. Assim, acredita-se que o uso de LMR como um marcador de prognóstico seria ser econômico e conveniente para o câncer de mama pacientes.

\section{Considerações Finais}

Em resumo, a relação das células pró-inflamatórias com os linfócitos possui um grande impacto no prognóstico de pacientes com câncer de mama. A NLR demonstrou um importante impacto na sobrevida, uma vez altas taxas de NLR estão associadas à uma menor taxa de sobrevida geral. Aliado à isso, altos valores de PLR foram identificados em pacientes com câncer metastático, enquanto baixos valores de LMR estão associado a um prognóstico positivo.

A análise dessas relações possui uma grande aplicabilidade para a prática clínica, pois parâmetros comuns do sangue periférico poderão presumir uma boa perspectiva de prognóstico em pacientes com câncer de mama. Ainda que este presente estudo tenha revisado o impacto desses biomarcadores inflamatórios no câncer de mama, trata-se de um tema relativamente recente e que precisa ser estudado mais afundo.

\section{Referências}

Abdalla, D. R., Simoens, C., Bogers, J. P., Murta, E. F. \& Michelin, M. A. (2015). Angiogenesis Markers in Gynecological Tumors and Patents for AntiAngiogenic Approach: Review. Recent Pat Anticancer Drug Discov. 2015,10(3):298-307.

Araújo, B. R. A., Coelho, C. A. D., Santos, J. O., Oliveira, M. de, Queiroz, R. de C. M., \& Abdalla, D. R. (2021). Papel da relação neutrófilo-linfócito no valor prognóstico para exacerbação e mortalidade entre indivíduos com Doença Pulmonar Obstrutiva Crônica: revisão integrativa da literatura. Research, Society and Development, 10(12), e86101220080.

Azambuja, E. (2007). Marcadores prognósticos e preditivos e sua importância na individualização do tratamento de pacientes com câncer de mama [Tese de Doutorado]. Universidade Federal Do Rio Grande do Sul. 
Batschauer, A. P. B., Fernandes, A. P. S. M. \& Carvalho, M. G. (2009). Avaliação hemostática e molecular em mulheres com câncer de mama receptor hormonal negativo. [Tese de Doutorado]: Universidade Federal de Minas Gerais.

Bianco, T. M., Abdalla, D. R., Desidério, C. S., et al. (20017). The influence of physical activity in the anti-tumor immune response in experimental breast tumor. Immunol Lett. 2017 Oct, 190:148-158.

Botelho, L. L. R., Cunha, C. C. A. \& Macedo, M. (2011). The integrative review method in organizational studies. Gestão e sociedade. 5(11): $121-136$.

Brasil. (2020). Ministério da Saúde. Instituto Nacional de Câncer. Estimativa: Incidência de câncer no Brasil. INCA, 2020.

Cho, U., et al. (2018). Prognostic value of systemic inflammatory markers and development of a nomogram in breast cancer. Plos One. $13(7)$ : e0200936.

Diana, C. A. F., et al. (2004). Factores pronósticos del cáncer de mama: modelo predictivo. Rev Oncol. 6(8):472-482.

Faria, E. H., Kim, D., Sisconetto, R. M., et al. (2021). Analysis of Knowledge About Male Breast Cancer Among Higher Education Male Students. Eur J Breast Health. 17(4):333-340.

Godinho, E. R. \& Koch, H. A. (2004). Breast cancer screening: physicians related issues. Radiol Bras. 37(2): 91-99.

Guerra, M. R. (2007). Sobrevida e fatores prognósticos para o câncer de mama em Juiz de Fora, Minas Gerais, na coorte diagnosticada entre 1998 e 2000. 2007. [Tese de Doutorado]. Instituto de Medicina Social, Universidade do Estado do Rio de Janeiro, Rio de Janeiro.

Huszno, J. \& Kolosza, Z. (2019). Prognostic value of the neutrophil lymphocyte, platelet lymphocyte and monocyte lymphocyte ratio in breast cancer patients. Oncol Lett. (6):6275-6283.

Huszno, J., et al. (2020). Prognostic Value of the Neutrophil-Lymphocyte, Platelet-Lymphocyte, and Monocyte-Lymphocyte Ratios in Male Breast Cancer Patients. Oncology. 98(7): 487-492.

Inumaru L., Silveira, E. A. \& Naves, M. M. V. (2011). Fatores de risco e de proteção para câncer de mama: uma revisão sistemática. Cadernos de Saúde Pública. 27(7): 1249-1270.

Losada B., et al. (2019) Pretreatment neutrophil/lymphocyte, platelet/lymphocyte, lymphocyte/monocyte, and neutrophil/monocyte ratios and outcome in elderly breast cancer patients. Clin Transl Oncol. 21(7):855-863.

Luchs, A. \& Pantaleão, C. (2010). Apoptose e modelos in vivo para estudo das moléculas relacionadas a este fenômeno. Einstein. 8(4): 495-497.

Ma, Y., Zhang, J. \& Chen, X., et al (2021). Lymphocyte-to-Monocyte Ratio is Associated with the Poor Prognosis of Breast Cancer Patients Receiving Neoadjuvant Chemotherapy. Cancer Manag Res. 13:1571-1580.

Nastri, C. O., Martins, W. P. \& Lenharte, R. J. (2011). Ultrasonography in breast cancer screening. Revista Femina. 39(2): 97-102.

Ribeiro-Silva, A. (2012). Core biopsy: uma técnica confiável para o diagnóstico histopatológico do câncer de mama? J Bras Patol Med Lab. 48(1): 8-9.

Salimena, A. M. O., Campos, T. S., Melo, M. C. S. C. \& Magacho, E. J. C. (2012). Mulheres Enfrentando O Câncer De Mama. remE - Rev. Min. Enferm.,16(3): 339-347.

Souza, M. T., Silva, M. D., \& Carvalho, R. (2010). Revisão integrativa: o que é e como fazer. Einstein, 8(1): $102-106$.

Van Berckelaer C., et al. (2020). A high neutrophil-lymphocyte ratio and platelet-lymphocyte ratio are associated with a worse outcome in inflammatory breast cancer. The Breast. 53: 212-220. 